\title{
$H$. pylori infection and the development of gastric cancer
}

\author{
Naomi Uemura, ${ }^{1}$ Shiro Okamoto ${ }^{2}$ and Soichiro Yamamoto ${ }^{2}$ \\ ${ }^{1}$ Department of Gastroenterology, International Medical Center of Japan, \\ ${ }^{2}$ Department of Gastroenterology, Kure Kyosai Hospital, Tokyo, Japan
}

\begin{abstract}
BACKGROUND: Recently, many study have shown that Helicobacter pylori infection is crucial in development of atrophic gastritis, which is closely associated with gastric cancer. We conducted a long-term endoscopic prospective follow-up study to investigate the development of gastric cancer in $H$. pylori-positive and -negative patients. METHODS: 1603 patients who underwent endoscopy and were assessed as to the presence of $H$. pylori infection by histology, rapid urease test and serologic test between April 1990 and March 1993 were entered. We prospectively studied 1246 subjects with and 280 subjects without $H$. pylori infection for a mean follow-up of 7.8 years (range 1-10.6 years). RESULTS: Gastric cancer of both the intestinal and diffuse type developed in $36(2.9 \%)$ infected patients but in none of the uninfected patients during follow-up. There was an increased risk for gastric cancer in infected patients with severe gastric atrophy and corpus predominant gastritis and intestinal metaplasia. Gastric cancer was detected in $21(4.7 \%)$ of the patients with non ulcer dyspepsia, in $10(3.4 \%)$ of those with gastric ulcer and in $5(2.2 \%)$ of those with gastric hyperplastic polyp, at enrollment. No gastric cancer was detected in duodenal ulcer patients. CONCLUSION: These results suggest that the development of both types of gastric cancer is caused by $\mathrm{H}$. pylori-associated gastritis, and the risk for development of gastric cancer in $\boldsymbol{H}$. pylori-negative subjects is extremely low. Subjects having $H$. pylori-positive gastric mucosa with severe atrophy and/or corpus gastritis may be at particularly high risk for gastric cancer.
\end{abstract}

Key words: Helicobacter pylori, gastric cancer, gastric mucosal atrophy, corpus gastritis

\section{Introduction}

Since the discovery of Helicobacter pylori ( $H$. pylori $),{ }^{1}$ the diagnosis and treatment of upper gastrointestinal disease has changed greatly. In 1991, three nested serological follow-up studies ${ }^{2-4}$ reported a higher risk for the development of gastric cancer in subjects serologically positive for $H$. pylori infection. The WHO/IARC consensus group ${ }^{5}$ stated in 1994 that there is sufficient epidemiological and histological ${ }^{6,7}$ evidence in humans to classify $H$. pylori as a Type- 1 carcinogen. Although the majority of recent studies have associated $H$. pylori with gastric cancer, there have been some contrary reports. ${ }^{8.9}$ The infection rates in gastric cancer patients vary greatly between different reports. These variations may be attributable to differences in the methods for detecting $H$. pylori in addition to differences in the patients' populations. Most prospective studies ${ }^{8,9}$ have used internal controls 'nested' within cohorts from which blood samples were taken some years before the onset of clinical gastric cancer. False negative cases are not uncommon, however, with various $H$. pylori diagnostic tests. ${ }^{10.11}$ Stringent investigation eliminating false negatives by several different tests is needed for the true assessment of $H$. pylori infection. ${ }^{12}$ No prospective study has assessed uninfected and infected patients using both invasive and non-invasive tests. In Japan where the incidence of gastric cancer is high, initial early-stage gastric cancer is often discovered by endoscopy. Under a significantly different medical insurance system from that in western countries endoscopy is performed inexpensively and frequently for the early detection of gastric cancer even for medical examination in asymptomatic patients, resulting in higher detection of early gastric cancer. However, $H$. pylori eradication therapy for the purpose of prevention of the cancer development is not yet approved because of a lack of clinical evidence.

Based on the above background, we conducted a prospective long-term endoscopic follow-up of a large group of patients, whose $H$. pylori status was stringently assessed by histological examination, rapid urease test with biopsy specimens and serological testing in order to determine the relationship between the development

Reprint requests to: Dr. Naomi Uemura, Department of Gastroenterology, International Medical Center of Japan, 1-21-1, Toyama, Shinjyuku-ku, Tokyo, Japan, e-mail: nuemura@imcj.hosp.go.jp 
of gastric cancer and $H$. pylori infection. We discuss the results in this study which were pblished elsewhere. ${ }^{13}$

\section{Patients and Methods}

\section{Patients and procedures}

Between April 1990 and March 1993, 1603 consecutive patients diagnosed as active duodenal ulcer (DU), active gastric ulcer (GU), gastric hyperplastic polyp (GP) and no findings (non ulcer dyspepsia: NUD) by endoscopy were enrolled in a prospective study. They were assessed for $H$. pylori status and followed-up endoscopically for early detection of gastric cancer. Patients with severe underlying diseases including gastric cancer, adenoma or those with gastric resection and subjects who had continuously received non-steroidal anti-inflammatory drugs (NSAIDs) were excluded. Seventy seven patients who refused a second endoscopic examination were excluded. The remaining 1524 patients ( 869 males and 657 females; $20-76$ years old, mean age 52 years old) were analyzed in the study. Endoscopies with biopsies were performed in all patients at enrollment and every 1 and 3 years. Follow-up was censored due to deaths from other causes and use of antibiotic treatment for $H$. pylori eradication. The mean duration of follow-up was 7.8 years (range 1-10.6 years). All patients gave written informed consent.

\section{Endoscopy and Histological examination}

All endoscopic examinations were performed with only local anesthesia (lidocaine). An Olympus videoscope (model GIF-230) was used. Four biopsy specimens were taken, 2 each from the greater curvature of the antrum and from the upper body of the stomach. Of these 4 specimens, 2 were fixed in formalin and assessed for $H$. pylori (Giemsa staining) and the degree of neutrophil infiltration and intestinal metaplasia (haematoxylin eosin staining). The remaining 2 were used for rapid urease test (CLO). The degree of neutrophil infiltration was classified into 4 grades (none: 0 , mild: 1 , moderate: 2, and marked: 3 ) and expressed as a score according to the Updated Sydney System. ${ }^{1+}$ Consensus was reached through revision of all the slides by the pathologists, leading to final conclusive scores. Subsequently, distribution pattern of active gastritis was classified into 4 types (no gastritis, antral predominant gastritis, and pangastritis and corpus predominant gastritis). The degree of intestinal metaplasia was classified into 2 grades (absence or presence) because of the multifocal distribution of metaplasia may lead to selection bias in only two biopsy specimens. Since only two biopsy were taken, gastric mucosal atrophy was evaluated according to the endoscopic atrophy border scale described by Kimura and Takemoto, ${ }^{15}$ which is well known to correlate with histological evaluation from previous reports. ${ }^{16.17}$ The grade was classified into 3 levels (none or mild, 1; moderate, 2; severe, 3 ). The pathologists were not aware of the clinical or endoscopic data and the results were scored blindly with the use of patient codes.

Rapid urease test (CLO test) was monitored for color change for up to 24 hours. Gastric cancer was defined as evident invasion of neoplastic epithelium into the lamina propria of the mucosa or beyond, i.e. category 5.1 or 5.2 according to the Vienna classification $^{18}$ and was classified according to Lauren ${ }^{19}$ into intestinal or diffuse type.

\section{Serological assay}

Blood was sampled immediately before endoscopy, and without delay, serum was separated therefrom, and cryopreserved at $-20^{\circ} \mathrm{C}$ until it was subjected to assay of antibody titer in serum (HM-CAP, Enteric Products, Westbury, NY) in accordance with the manufacturer's instructions. In the determination, a cut off value was set to a titer of 1.8 , and a titer of equal to or more than 1.8 was determined as $H$. pylori positive.

\section{Determination of $H$. pylori infection}

The presence of $H$. pylori infection was determined by histological examination, rapid urease test and serological evaluation. Patients positive on histological examination and/or rapid urease test and/or serological search were considered $H$. pylori-positive, while those in whom all three assays were negative were considered H. pylori-negative.

\section{Statistical analysis}

All statistical analysis was performed with the SAS software. ${ }^{20}$ The demographic and clinical characteristics of the patients were compared statistically by the Student t-test (age, duration of follow up, number of endoscopies) or Chi-squared test (sex, diagnosis, grading of gastric mucosal atrophy, distribution of gastritis, intestinal metaplasia). The statistical calculation on the relation between gastric cancer development and gastric findings such as degree of the atrophy, pattern of gastritis distribution and presence of intestinal metaplasia was able to calculate the Relative Risks (RR) with the Cox proportional Hazards models. Since the development of the gastric cancer has not been recognized to the $H$. pylori-negative patients and duodenal ulcer patients and patients eradicated $H$. pylori, it was impossible to calculate the difference in the incidence of the gastric cancer by Cox proportional Hazards. For 
this reason, A Kaplan-Meier curve analysis and Chisquared test or Fischer extract method were used for the difference in proportion in those cases. All $\mathrm{P}$ Values are for two-tailed comparisons. Values were considered significant at a $5 \%$ probability level.

\section{Results and Discussion}

The comparison of the demographic and clinical characteristics of $H$. pylori-positive and -negative patients and The development of gastric cancer during follow-up in each group

All 1526 patients were classified for $H$. pylori status 280-negative and 1246-positive. There were no differences in age, gender or mean number of endoscopies. H. pylori-positive patients included 445 with NUD (206 males and 239 females; 22-76 years old, mean age 54 years old), 275 with DU (male: 198, female: $77 ; 48 \mathrm{y}$ ), 297 with GU (male: 226, female: 71; 52 y) and 229 with GP (male: 84 , female: $145 ; 56$ y). $H$. pylori-negative patients in the study included only NUD. Atrophy and intestinal metaplasia of any grade was found in only $4 \%$ and $2 \%$ of the uninfected group at enrolment and only $2 \%$ had gastritis, all antral predominant. On the other hand, in the infected group moderate atrophy was found in $53 \%$, and severe atrophy in $17 \%$. Antral predominant gastritis was found in $56 \%$, pangastritis in $27 \%$ and corpus predominant in $17 \% .37 \%$ had intestinal metaplasia. There were significant differences in these parameters between both groups $(\mathrm{P}<0.0001$ by Chi-squared test). Duration of follow-up in infected group was significantly shorter than that in uninfected group $(P=0.0008)$, because 253 of 1256 infected patients received eradication therapy at an early stage of follow-up.

The development of gastric cancer during follow-up in each group is shown in Table 1. Gastric cancer of both the intestinal and diffuse type developed in 36 $(2.9 \%)$ infected patients but in none of the uninfected patients during follow-up. Because no gastric cancer

Table 1 The Development of Gastric Cancer during Follow-up in H. pylori-positive and -negative Patients

\begin{tabular}{lll}
\hline \hline Total subjects & 1526 cases $(1990 / 4-1993 / 3)$ \\
H. pylori status & $\begin{array}{l}\text { positive } \\
(\mathrm{n}=1246)\end{array}$ & $\begin{array}{l}\text { negative } \\
(\mathrm{n}=280)\end{array}$ \\
& & $\downarrow$ \\
subsequent gastric cancer & $36(2.9 \%)^{*}$ & $0(0 \%)$ \\
intestinal type & 23 & 0 \\
diffuse type & 13 & 0 \\
\hline
\end{tabular}

$*: P=0.004$ (chi-square test) vs: negative group. developed in any of the uninfected group, their Relative Risk could be determined. The rate of development of cancer was significantly higher in positives than $H$. pylori-negative patients by Fischer extract method $(\mathrm{P}<0.0001)$. The 36 patients with gastric cancer comprised 23 males and 13 females; (mean age 60 years old, range $41-76$ y at baseline, mean age 65 y $47-83$ y at the detection of gastric cancer). Histology revealed 23 intestinal type (16 males and 7 females; mean age $64 \mathrm{y}$, $44-76$ y at baseline, 53-83, mean age 70 y at the detection of gastric cancer) and 13 diffuse type (6 males and 7 females; mean age $52 \mathrm{y}, 41-68$ y at baseline, 47-75, mean age $58 \mathrm{y}$ at the detection of gastric cancer). Patients' mean age at enrolment and at the time of detection of gastric cancer was significantly younger in the diffuse type than the intestinal type $(\mathrm{P}<0.0001)$.

This prospective follow-up of a large series of patients aimed to detect early gastric cancer by endoscopy after evaluation of $H$. pylori infection using biopsy and serology. During the observation period, gastric cancer appeared only in patients with $H$. pylori infection and not in uninfected patients. The results of this study support a recent meta-analysis reported by Huang et al. ${ }^{8}$ which showed that $H$. pylori infection was epidemiologically involved in the onset of gastric cancer. In Japan, it has been reported that 300,000 individuals or $0.5 \%$ of the 60 million who are $H$. pylori positive develop gastric cancer annually, ${ }^{21}$ which means that $5 \%$ of $H$. pylori positive individuals develop gastric cancer every 10 years. The results of the present study support this estimate and it is clear that the risk of gastric cancer developing in $H$. pylori carriers is extremely high in Japan.

This study also shows that it is very rare for individuals without $H$. pylori infection to develop gastric cancer. In many previous epidemiological studies showing a close relationship between $H$. pylori infection and gastric cancer, a large number of $H$. pylori serologically negative patients were found to have cancer ${ }^{8.9}$ Recent studies ${ }^{10.11}$ have shown that false-negative results occur using the serum antibody method, so it is possible that the $H$. pylori positive rate has been underestimated in gastric cancer patients. Tabata et al. ${ }^{12}$ studied the $H$. pylori positive rate in gastric cancer patients using various methods and concluded that false-negative results occur with the serum antibody method. They also stated that a biopsy from the greater curvature of the upper gastric body should be taken because this results in fewer false negatives. Enomoto et al. ${ }^{22}$ performed an immunohistological study taking biopsy specimens from the greater curvature of the upper gastric body and $H$. pylori antibodies, and found that the $H$. pylori positive rate was $98 \%$ in gastric cancer patients. Their results and our findings suggest that when $H$. pylori infection is carefully sought, there are very few $H$. pylori negative 
Table 2 The Baseline Abnormalities of the Gastric Mucosa in Both Groups

\begin{tabular}{lccc}
\hline & $\begin{array}{l}\text { H. pylori- } \\
\text { positive } \\
(\mathrm{n}=1246)\end{array}$ & $\begin{array}{l}\text { H. pylori- } \\
\text { negative } \\
(\mathrm{n}=280)\end{array}$ & P-value \\
\hline 1) grading of gastric atrophy & & & $<0.0001$ \\
$\begin{array}{l}\text { non-mild (\%) } \\
\text { moderate (\%) }\end{array}$ & $381(30)$ & $270(96)$ & \\
severe (\%) & $657(53)$ & $3(1)$ & \\
2) distribution of gastritis & $208(17)$ & $7(3)$ & \\
non gastritis (\%) & 0 & & $<0.0001$ \\
$\begin{array}{l}\text { antral predominant (\%) } \\
\text { pangastritis (\%) }\end{array}$ & $699(56)$ & $6(2)$ & \\
corpus predominant (\%) & $337(27)$ & $0(0)$ & \\
3) intestinal metaplasia & $210(17)$ & $0(0)$ & \\
positive (\%) & $464(37)$ & $5(2)$ & $<0.0001$ \\
\hline
\end{tabular}

gastric cancer patients. In $H$. pylori negative patients, it is rare to find histological gastritis, especially neutrophil infiltration, and it is well known that little gastric mucosal atrophy occurs. ${ }^{23,24}$ The same results were obtained in this study. Thus, the onset of gastric cancer is based on histological gastritis or atrophic gastritis associated with $H$. pylori infection.

\section{The increased risk for gastric cancer in infected patients}

The background gastric mucosa at baseline in all infected patients and in the 36 gastric cancer patients is shown in Table 2. Gastric cancer developed in $3(0.8 \%)$ of 381 patients with no or mild atrophy, $18(2.7 \%)$ of 657 with moderate atrophy and $15(7.2 \%)$ of 208 with severe atrophy. Gastric cancer developed in $2(0.3 \%)$ out of 699 patients who had antral predominant gastritis at the start of follow-up, 14(4.2\%) of 337 with pangastritis and $20(9.5 \%)$ of 210 with corpus predominant gastritis. Gastric cancer developed in $5(0.6 \%)$ of 782 patients without intestinal metaplasia, $26(5.6 \%)$ of 464 with intestinal metaplasia. An increased risk for gastric cancer in infected patients with moderate or severe atrophy (Relative Risk (RR) and $95 \%$ confidence interval of the difference: CI with no or mild atrophy: 2.8 or $4.9,1.5-5.2$ or $2.8-19.2)$, and pangastritis or corpus predominant gastritis (RR, 95\% CI with antral predominant pattern: 15.6 or $34.5,6.5-36.8$ or $7.1-166.7$ ) and presence of intestinal metaplasia (RR, $95 \% \mathrm{CI}$ with no intestinal metaplasia: $6.5,2.6-16.1$ ).

The frequency of severe atrophy, corpus predominant gastritis and presence of intestinal metaplasia was significantly higher in patients with intestinal type than in diffuse type gastric cancer $(P=0.002,<0001$ and $=0.0083$ by Fischer extract method). Notably, in 14 patients with diffuse type nine had moderate atrophy and 10 had pangastritis.
These results suggested that $H$. pylori positive patients with severe atrophic gastritis and/or corpuspredominant gastritis accompanying intestinal metaplasia have a high risk of developing intestinal type gastric cancer. It has long been known that intestinal type gastric cancer occurs in patients who have severe atrophic gastritis associated with intestinal metaplasia. ${ }^{25}$ It is also well known that progression of atrophic gastritis can be caused by $H$. pylori infection. ${ }^{26}$ Our results reconfirm the hypothesis of Correa ${ }^{25}$ that severe atrophic gastritis accompanying intestinal metaplasia caused by persistent $H$. pylori infection is closely related to the development of intestinal type gastric cancer. Since atrophic changes are not severe in diffuse type gastric cancer, ${ }^{26,27}$ it was previously considered to have little relationship with $H$. pylori infection. However, recent epidemiological and histopathological studies $^{28,29}$ have shown that the development of diffuse type cancer is also closely related to $H$. pylori. In the present study, many of the diffuse type gastric cancer patients showed moderate atrophic changes and pangastritis. Our results support the hypothesis of Sipponen et al. ${ }^{26}$ and Solcia et $a l^{27}$ that diffuse type gastric cancer occurs during the progression of atrophic gastritis in patients with $H$. pylori infection and is associated particularly with active gastritis.

The development of gastric cancer in various diseases in H. pylori-positive patients (Table 3)

Gastric cancer was detected in $21(4.7 \%)$ of the patients with NUD, in $10(3.4 \%)$ of those with GU and in $5(2.2 \%)$ of those with GP at baseline. No gastric cancer was detected in DU patients. The frequency of gastric cancer development in NUD, GU and GP patients was significantly higher than that in DU patients $(\mathrm{P}=0.0028, \mathrm{P}=0.0106$ and $\mathrm{P}=0.0448$ by Fischer extract method). Notably, the frequency of diffuse type cancer in GU was significantly higher than that in NUD and GP (P $=0.0315$ by Chi-squared test $)$ and the mean age (52.7 y) of gastric cancer in GU was significantly younger than that in NUD $(62.6 y)(P=0.0087$ by $\mathrm{t}-$ test).

Table 3 Endoscopic Findings and the Development of Gastric Cancer

\begin{tabular}{lcccc}
\hline \hline Endoscopic finding & Cases & Cancer (\%) & \multicolumn{2}{c}{ Histology } \\
\cline { 4 - 5 } & & & Intestinal & Diffuse \\
\hline active duodenal ulcer & 275 & $0(0)$ & 0 & 0 \\
hyperplastic polyp & 229 & $5(2.2)$ & 4 & 1 \\
active gastric ulcer & 297 & $10(3.4)$ & 3 & 7 \\
non ulcer dyspepsia & 445 & $21(4.7)$ & 16 & 5 \\
\hline
\end{tabular}


In this prospective study, gastric cancer developed in patients with non ulcer dyspepsia, active gastric ulcer and hyperplastic gastric polyps, but no gastric cancer occurred during follow-up in patients with active duodenal ulcer. A large-scale controlled study by Hansen et al. ${ }^{30}$ has shown that gastric ulcer is associated with a high risk of gastric cancer; while duodenal ulcer has a low risk of cancer. Our results agree with those of Hansen et al. It is known that atrophic gastritis and corpus-predominant gastritis characterize the background mucosa of gastric ulcer patients, while duodenal ulcer patients show little atrophic change and have antral-predominant gastritis. $^{31^{-33}}$ Therefore, there should be more gastric cancer in patients with gastric ulcers than in those with duodenal ulcers. Gastric ulcer patients show a strong predominance of diffuse type gastric cancer, and many are relatively young. In young patients with gastric ulcer, it is therefore necessary to perform careful follow-up to detect diffuse type gastric cancer even after ulcer healing.

\section{Effect of eradication on the development of gastric cancer}

253 of infected patients received eradication therapy. Although they were treated as censored cases, were also followed up after therapy. No gastric cancer developed after eradication in these patients. Mean length of follow-up after eradication $(4.8 \pm 1.2$ years) was significantly shorter than that of no treatment patients $(8.5 \pm 1.7$ years $)(\mathrm{P}<0.0001)$

Thus no gastric cancer developed after eradication in 253 infected patients in this study, although duration of follow-up was relatively short. We have previously shown that in patients with early gastric cancer treated by endoscopic mucosal resection (EMR) eradication of $H$. pylori prevents the development of new cancer or the continued growth of occult cancer. ${ }^{34}$ Shimizu et al. reported that eradication reduced the risk of development of gastric cancer in Mongolia gerbils. ${ }^{35} \mathrm{We}$ strongly recommend that $H$. pylori eradication therapy should be performed not only to prevent ulcer relapse, but also to prevent gastric cancer.

In conclusion, the development of both intestinal and diffuse gastric cancer is caused by $H$. pylori-associated gastritis. $H$. pylori-positive gastric mucosa with severe atrophy accompanying intestinal metaplasia and/or corpus predominant gastritis are at a particularly high risk in Japan.

\section{References}

1. Warren JR, Marshall B: Unidentifid curved bacilli on gastric epithelium in active chronic gastritis. Lancet 1983; 1: 1273-1275
2. Parsonnet J, Friedman GD, Daniel MS, Vandersteen DP, Chang Y, Vogelman JH, Orentreich N, Sibley RK: Helicobacter pylori infection and the risk of gastric carcinoma. N Engl J Med 1991; 325: $1127-31$

3. Nomura A, Stemmermann GN, Chyouph, Kato I, Perez-Perz GI, Blaser MJ: Helicobacter pylori infection and Gastric carcinoma among Japanese Americans in Hawaii. N Engl J Med 1991: 325: $1132-6$

4. Forman D, Newell DG, Fullerton F, Yarnell JW, Stacey AR, Wald N, Sitas F: Association between infection with Helicobacter pylori and risk of gastric cancer: evidence from prospective investigation. Br Med J 1991; 302: 1302-1305

5. IARC Working Group. Monographs on the evaluation of the carcinogenic risks to humans: schstsomes, liver flukes and Helicobacter pylori. IARC Publications Geneva 1994; 177-241

6. Correa P, Fox J, Fontham E, Ruiz B, Lin Y, Zavala D, Taylor M, Mackenley D, Lima E, Portilla H, Zarama G: Helicobacter pylori and gastric carcinoma. Serum antibody prevalence in populations with contrasting cancer risks. Cancer 1990; 66: 25692574

7. Sipponen $\mathrm{P}$, Hyvarinen $\mathrm{H}$ : Role of Helicobacter pylori in the pathogenesis of gastritis, peptic ulcer and gastric cancer. Scand $\mathbf{J}$ Gastroenterol 1993; 196: 3-6

8. Huang JQ, Sridhar S, Chen Y, Hunt R: Meta-analysis of the relationship between Helicobacter pylori seropositivity and gastric cancer. Gastroenterology 1999; 114: 1169-1179

9. Danesh J: Helicobacter pylori infection and gastric cancer: systematic review of the epidemiological studies. Aliment Pharmacol Ther 1999; 13: 851-856

10. Ekstrum AM, Held M, Hansson L: Helicobacter pylori in gastric cancer established by CagA immunoblot as a marker of past infection. Gastroenterology 2000; 121: 784-791

11. Maeda S, Yoshida H, Ogura K, Yamaji Y, Ikenoue T, Mitsushima T, Tagawa H, Kawaguchi R, Mori K, Mafune K, Kawabe T, Shiratori $\mathrm{Y}$, Omata $\mathrm{M}$ : Assessment of gastric carcinoma risk associated with Helicobacter pylori may vary depending on the antigen used: CagA specific enzyme-linked immunoadsorbent assay (ELISA) versus commercially available $H$. pylori ELISAs. Cancer 2000; 88: 1530-1535

12. Tabata H, Fuchigami T, Kobayashi H, Sakai Y, Nakanishi M, Tomioka K, Nakamura S, Fujishima M: Helicobacter pylori and mucosal atrophy in patients with gastric cancer: A special study regarding the methods for detecting Helicobacter pylori. Dig Dis Sci 1999; 44: 2027-2034

13. Uemura N, Okamoto S, Yamamoto S, Matsumura N, Yamaguchi S, Yamakido M, Taniyama K, Sasaki N, Schlemper RJ: Helicobacter pylori infection and the development of a gastric cancer. N Eng J Med 2001; 345: 784-789

14. Dixon MF, Genta RM, Yardley JH, Correa P: Classification and grading of gastritis: the updated Sydney System. Am J Sur Pathol 1996; 20: 1161-1181

15. Kimura K, Takemoto T: Endoscopic atrophy border. Endoscopy 1969; 1: 1-3

16. Satoh K, Kimura K, Taniguchi Y, Yoshida Y, Kihira K, Takimoto T, Kawata H, Saifuku K, Ido K, Takemoto T, Ota Y, Tada M, Karita M, Sakaki N, Hoshihara Y: Distribution of inflammation and atrophy in the stomach of Helicobacter pylori-positive and -negative patients with chronic gastritis. Am J Gastroenterol 1996; 91: 963-969

17. Ito S, Azuma T, Murakita H: Profile of Helicobacter pylori cytotoxin derived from two areas of Japan with different prevalence of atrophic gastritis. Gut 1996; 39: 800-6

18. Schlemper RJ, Riddell RH, Kato Y, Borchard F. Cooper HS, Dawsey SM, Dixon MF, Fenoglio-Preiser CM, Flejou JF, Geboes K, Hattori T, Hirota T, Itabashi M, Iwafuchi M, Iwashita A, Kim YI, Kirchner T, Klimpfinger M, Koike M, Lauwers GY. 
Lewin KJ, Oberhuber G, Offner F, Price AB, Rubio CA, Shimizu M, Shimoda T, Sipponen P, Solcia E, Stolte M, Watanabe $\mathrm{H}$, Yamabe $\mathrm{H}$ : The Vienna classification of gastrointestinal epithelial neoplasia. Gut 2000; 47: 251-255

19. Lauren P: The two histological main types of gastric carcinoma: diffuse and so called intestinal type. Acta Pathol Microbiol Scand (A) 1965; 64: 31-49

20. SAS/STAT software: changes and enhancement, through release 6.11. Cary, N.C. SAS Institute, 1996

21. Asaka M: Gastric cancer: Helicobacter pylori and gastroduodenal diseases. Asaka M, ed. Sentan Igakusha. Tokyo 1999. pp. 116-126 (in Japanese)

22. Enomoto $H$, Watanabe $H$, Nishikura $K$, Umezawa $H$, Asakura H: Topographic distribution of Helicobacter pylori in the resected stomach. Eur J Gastroenterol Hepatol 1998; 10: 473-478

23. Blaser MJ: Hypothesis on the pathogenesis and natural history of Helicobacter pylori-induced inflammation. Gastroenterology 1992; 102: 720-727

24. Kuipers EJ, Uyterlinde AM, Pena AS, et al: Long-term sequence of Helicobacter pylori gastritis. Lancet 1995 ; 345: 1525-1528

25. Correa P: Human gastric carcinogenesis: a multistep and multifactorial process. Cancer Res 1992: 52: 6735-6740

26. Sipponen M. Kosunen TU, Valle J, Riihla M, Seppala K: Helicobacter pylori infection and chronic gastritis in gastric cancer. $\mathrm{J}$ Clin Pathol 1992; 45: 319-323

27. Sorcia E, Fiocca R, Luinetti O: Intestinal and diffuse gastric cancers arise in a different background of Helicobacter pylori gastritis through different gene involvement. Am J Surg Path 1996; 20(Suppl.1): s18-s22
28. Kikuchi S, Wada O, Nakajima T, Nishi T, Kobayashi O, Konishi $\mathrm{T}$, Inaba Y: Serum anti-Helicobacter pylori antibody and gastric carcinoma among young adults. Cancer 1995; 75: 2789-2793

29. Kokkola A, Valle J, Haapianen R, Sipponen P, Kivilaakso E, Puolakkainen P: Helicobacter pylori infection in young patients with gastric carcinoma. Scand J Gastroenterol 1996; 31: 643-647

30. Hansson LE, Nyren O, Hsing AW, Bergstrom R, Josefsson S Chow W-H, Fraumeni JF, Adami HO: The risk of stomach cancer in patients with gastric or duodenal ulcer disease. $\mathrm{N}$ Engl $\mathrm{J}$ Med 1996; 335: 242-249

31. Stolte M, Eidt S, Ohsmann A: A difference in Helicobacter pylori-associated gastritis in the antrum and the body of the stomach. Z Gastroenterol 1990; 28: 229-233

32. Meining A, Stolte M, Hatz R, Lehn N, Miehlke S, Morgner A, Bayerdorffer E: Differing degree and distribution of gastritis in Helicobacter pylori-associated diseases. Vircow Arch 1997; 431: $11-15$

33. Graham DY: Helicobacter pylori: its epidemiology and its role in duodenal ulcer disease. J Gastroenterol Hepatol 1991; 6: 105113

34. Uemura N, Mukai T, Okamoto S: Effect of Helicobacter pylori eradication on subsequent development of cancer after endoscopic resection of early gastric cancer. Cancer Epidemiol Biomarkers Prev 1997; 6: 639-642

35. Shimizu N, Ikehara $Y$, Inada K, Nakanishi $H$, Tsukamoto $T$, Nozaki K, Kaminishi M, Kuramoto S, Sugiyama A, Katsuyama $\mathrm{T}$, Tatematsu M: Eradication diminishes enhancing effects of Helicobacter pylori infection on glandular stomach carcinogenesis in Mongolian gerbils. Cancer Res 2000; 60: 1512-1514 